\title{
The Muon Puzzle in air showers and its connection to the
}

\section{LHC}

Johannes Albrecht, ${ }^{a}$ Lorenzo Cazon, ${ }^{b}$ Hans Dembinski, ${ }^{a, *}$ Anatoli Fedynitch, ${ }^{c}$ Karl-Heinz Kampert, ${ }^{d}$ Tanguy Pierog, ${ }^{e}$ Wolfgang Rhode, ${ }^{a}$ Dennis Soldin, ${ }^{f}$ Bernhard Spaan, ${ }^{a}$ Ralf Ulrich ${ }^{e}$ and Michael Unger ${ }^{e}$

${ }^{a}$ Faculty of Physics, TU Dortmund, Otto-Hahn Str. 4a, 44221, Dortmund, Germany

${ }^{b}$ Laboratorio de Instrumentacao e Fisica Experimental de Particulas (LIP), Av. Elias Garcia, 14-1, P-1000 Lisbon, Portugal

${ }^{c}$ Institute for Cosmic Ray Research, The University of Tokyo, 5-1-5 Kashiwa-no-ha, Kashiwa, Chiba 277-8582, Japan

${ }^{d}$ Department of Physics, Bergische Universität Wuppertal, Gaußstr. 20, 42119 Wuppertal, Germany

${ }^{e}$ Institute for Astroparticle Physics, Karlsruhe Institute of Technology (KIT), Hermann-von-Helmholtz-Platz 1, 76344 Eggenstein-Leopoldshafen, Germany

${ }^{f}$ Bartol Research Institute and Dept. of Physics and Astronomy, University of Delaware, 217 Sharp Laboratory, Newark, DE 19716, United States

E-mail: hans.dembinski@tu-dortmund.de

High-energy cosmic rays are observed indirectly by detecting the extensive air showers initiated in Earth's atmosphere. Air showers are hadronic cascades, which eventually decay into muons and the muon number is a key observable to infer the mass composition of cosmic rays. The interpretation of these observations relies on accurate models of air shower physics, which is a challenge and an opportunity to test QCD under extreme conditions. Air shower simulations with state-of-the-art QCD models show a significant muon deficit with respect to measurements; this is called the Muon Puzzle. The origin of this discrepancy has been traced to the composition of secondary particles in hadronic interactions. The muon discrepancy starts at the TeV scale in the centre-of-mass frame, which suggests that the origin should be observable at the Large Hadron Collider. An effect that can potentially explain the puzzle has been observed at the LHC, but needs to be confirmed with forward facing experiments, and with future data on oxygen beams.

$37^{\text {th }}$ International Cosmic Ray Conference (ICRC 2021)

July 12 th - 23rd, 2021

Online - Berlin, Germany

\footnotetext{
*Presenter
} 


\section{Introduction}

At cosmic ray energies above about $100 \mathrm{TeV}$, the flux of cosmic rays is so low that direct observation with satellite and balloon experiments becomes infeasible. At higher energies, cosmic rays are observed indirectly via air showers, hadronic cascades initiated by high-energy cosmic rays in the atmosphere. The established methods to observe air showers are the detection of charged particles which reach the ground with arrays of detectors and the optical observation of the emitted Cherenkov and fluorescence light in the atmosphere. Radio detection of air showers is a new observation method that combines advantages of optical and ground measurements.

The indirect observation requires accurate air shower simulations, since properties of the cosmic ray have to be inferred from observed features of the air shower. The cosmic ray community has build sophisticated simulation packages that integrate state-of-the-art models of electromagnetic and hadronic interactions. Air showers simulated with these programs describe real showers quite successfully, but there is a long-standing discrepancy between simulated and observed muon production in air showers.

Tensions between simulation and experiment are observed in several aspects of muon production. The most prominent and extensively studied deviation is that of the total number of muon $N_{\mu}$ which arrive at the ground. The muon abundance is significantly lower in simulations than in experiments. Air shower simulation codes use a variety of models for hadronic interactions, which all fail to reproduce the large number of muons observed in ultra-high energy air showers. This is a major obstacle for the field, since the muon number and the depth of shower maximum $X_{\max }$ are the two main features to infer the mass composition of cosmic rays as function of their energy.

The energy-dependent mass composition is very sensitive to properties of the dominant sources of cosmic rays and the propagation of cosmic rays through space, and therefore of great interest. The mass composition inferred from $X_{\max }$ is in the expected range between proton and iron, while the composition inferred from $N_{\mu}$ suggests a composition heavier than iron above $10 \mathrm{EeV}$. This is astrophysically not plausible. Furthermore, our current understanding of air shower physics suggests that the composition obtained from $X_{\max }$ is more likely to be accurate than the result obtained from $N_{\mu}$, as the underlying processes seem to be better understood. Nevertheless, the ambiguity casts a doubt on the interpretation of air shower data and hence it is very important to resolve it.

The muon discrepancy is interesting from the point of view of elemental particle physics, too, because it could indicate missing physics in current state-of-the-art models of hadronic interactions, which describe the hadronic cascade that leads up to the muon production. The dominant interactions in air showers have low momentum transfer and cannot be computed from first principles with perturbative quantum chromodynamics. Effective theories are used to describe these interactions, and it is possible that an important effect is missing in these theories.

In this light, the recently discovered multiplicity-dependent strangeness enhancement at midrapidity in proton-proton and proton-lead collisions at the LHC by the ALICE collaboration [1] is of special interest. This kind of strangeness enhancement was previously only observed in heavy-ion collisions, where the common model to interpret these data is based on the formation and subsequent freeze-out of a quark gluon plasma (QGP). The theory community is divided over the question whether a QGP can also form in proton-lead or proton-proton collisions.

Even though the mechanism that causes multiplicity-dependent strangeness enhancement is 
currently unclear, the effect phenomenologically changes the hadron composition in such a way that the muon number in air showers is increased. The phenomenology has been explored with a toy model $[2,3]$ with the conclusion that this effect could be the missing piece in the muon puzzle.

This proceeding is based on a recent review on the muon puzzle and its connection to the LHC [4], which summarizes the current knowledge about the muon discrepancy in air showers, the state of air shower simulations, what we have learned about the connection of microscopic hadronic physics and muon production in air showers and finally what we have already learned from the LHC and what kind of measurements are still needed. In the following sections we will briefly summarize the main arguments and refer the reader to the review for details.

\section{Meta-analysis of muon measurements in air showers}

There are several deviating aspects between the simulated muon component in air showers and measurements, which are listed in Ref. [4]. The most striking and best explored is the deviation in the mean number of $\mathrm{GeV}$ muons as observed by ground arrays of particle detectors. This quantity, or proxies thereof, have been measured by multiple experiments, with initially contradicting results. These apparent contradictions have been largely reduced by a meta-analysis from the Working group on Hadronic Interaction and Shower Physics (WHISP) which was formed in 2018 by members of eight (now nine) air shower experiments [5-7].

The working group addressed two challenges. Firstly, muon measurements were not directly comparable, since they were carried out at different distances to the shower axis, at different altitudes, at different zenith angles, and with different energy thresholds for muons. These aspects affect the measured muon number. To make the results comparable, the logarithmic $z$-scale was introduced,

$$
z=\frac{\ln N_{\mu}-\ln N_{\mu, p}}{\ln N_{\mu, \mathrm{Fe}}-\ln N_{\mu, p}},
$$

where $\ln N_{\mu}$ is the logarithm of the muon number (or its proxy) as measured by the detector, while $\ln N_{\mu, p}$ and $\ln N_{\mu, \mathrm{Fe}}$ are the corresponding simulated numbers (on detector-level) from an air shower simulation with a particular hadronic interaction model. The caveat of this approach is that measurements can only be compared if air shower simulations with the same hadronic interaction model exist. This makes it difficult to compare old results with the latest results. Secondly, the muon number scales almost linearly with the shower energy, which means that known systematic offsets in the energy scales of different experiments affects $z$, since the simulations are compared to the measurement at the energy that the experiment reports. If the energy scales of two experiments have a relative offset, then this offset translates into offsets in $z$. These relative offsets were corrected for the participating experiments by exploiting that the cosmic ray flux on Earth is the same everywhere, and by assuming that relative offsets in the apparent cosmic ray flux measured by each experiment originate solely from offsets in their energy scales. A correction of this effect significantly reduced the spread of $z$ values, as expected.

The energy-scale corrected $z$-values from nine experiments and for eight hadronic interaction models are shown in Fig. 1, more details on the latest update of the meta-analysis and further checks performed on the data are given in Ref. [7]. Above $10 \mathrm{EeV}$, the $z$-values exceed those of pure iron showers for all models, which is astrophysically not plausible. Furthermore, the values should be 


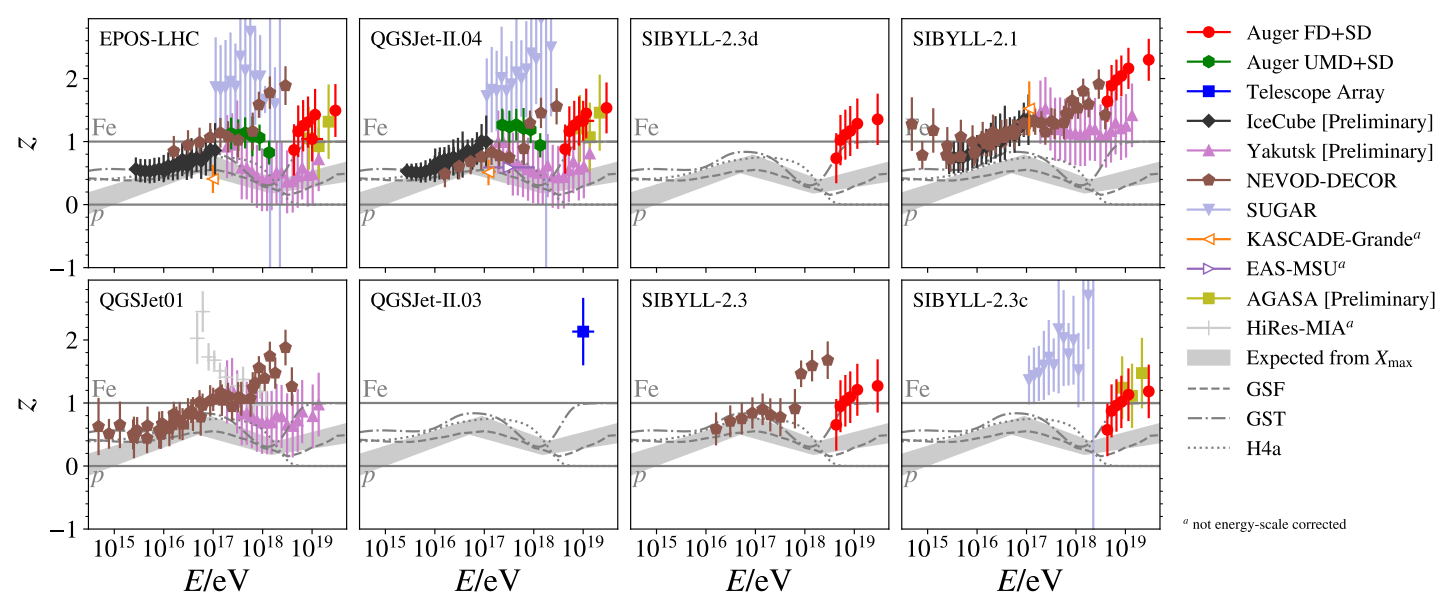

Figure 1: Measurements of muons produced in air showers from nine experiments after adjusting for energyscale offsets (points) converted to the $z$-scale as function of shower energy for different hadronic interaction models. Shown for reference are expected values from global fits to air shower data (lines) and from optical measurements of the depth of shower maximum (band). The image was taken from Ref. [7].

consistent with predictions based on optical measurements of the depth of shower maximum $X_{\max }$, but they exceed these predictions above $10^{17} \mathrm{eV}$.

Several conclusions can be drawn from these results. An experimental mistake as the origin of the discrepancy can be ruled out, since the effect is seen by several experiments, including those run by the largest collaborations with carefully evaluated systematic uncertainties. Potential mistakes in the air shower simulations codes can be ruled out, since several codes have been developed independently, and these codes have been compared. The muon number varies at the $5 \%$ level between codes, which is not enough to explain this effect [4]. A large mistake in the propagation of $\mathrm{GeV}$ muons in air can also be ruled out, although there are ongoing efforts to improve the precision for $\mathrm{TeV}$ muons propagating in dense media like water and ice [8-12], which will also benefit air shower simulation.

Progress has been made in recent years on the understanding of air shower physics and the possible microscopic origin of this discrepancy, which is discussed in the following. The discrepancy has an early onset, at about $4 \cdot 10^{16} \mathrm{eV}$, which corresponds to a centre-of-mass energy $\sqrt{s_{\mathrm{NN}}}=8 \mathrm{TeV}$ in the first interaction. This indicates that the origin of the discrepancy should be observable at the LHC $[2,4,13]$. Exotic explanations for the discrepancy are at tension with the early onset and with the first measurement of the muon number fluctuations [14] in ultra-high energy showers by the Pierre Auger Observatory, which are sensitive to exotic theories but found to be consistent with current hadronic interaction models.

\section{Muon production in air showers}

An air shower is a hadronic cascade in which the kinetic energy of the cosmic ray is successively converted into secondary particles in inelastic interactions with the atmosphere. The HeitlerMatthews model [15] is a simplified model of an air shower. A schema is shown in Fig. 2 on the 

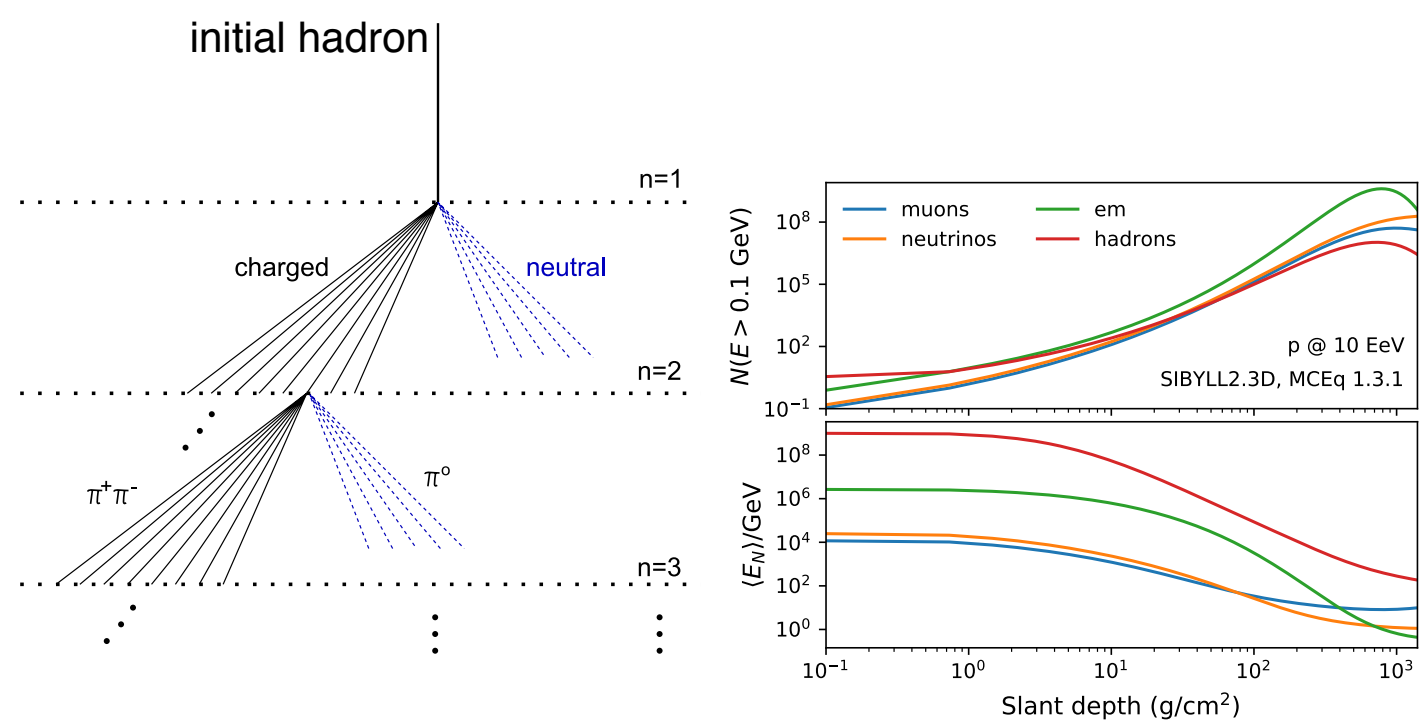

Figure 2: Left: Sketch of the Heitler-Matthews model [15] of an air shower initiated by a cosmic ray, in which only pions are produced. Image from Ref. [16]. Right: Number and average energy of different particle species in a proton air shower at $10 \mathrm{EeV}$ as a function of the traversed slant depth simulated with MCEq [12] and SIBYLL-2.3d [17]. Image from Ref. [4].

left-hand side. Only pions are produced in this model. Charged pions have long lifetimes and interact again to form a hadronic cascade. Neutral pions decay immediately into photons, which form a decoupled electromagnetic cascade. There is a small feedback from the electromagnetic into the hadronic cascade via photo-nuclear interactions, but this effect is neglected here.

The energy of the parent pion in the model is distributed equally onto its children in the model, which means that the pion energy decreases after each interaction. After $k$ steps of the cascade, the energy falls below the critical energy, where decay becomes more probable than another collision. At this point, the pions decay into muons.

The number of muons $N_{\mu}$ as a function of the energy $E$ and nuclear mass $A$ of the cosmic ray can be computed in this simplified model as

$$
N_{\mu}(E, A)=A^{(1-\beta)}\left(\frac{E}{\xi_{h}}\right)^{\beta} \text { with } \beta=\frac{\ln \left(\alpha N_{\text {mult }}\right)}{\ln N_{\text {mult }}},
$$

where $\alpha$ is the fraction of charged pions produced, $N_{\text {mult }}$ is the hadron multiplicity, and $\xi_{h}$ is the critical energy for pions. This functional relationship is also found in full air shower simulations, like those shown in Fig. 2 on the right-hand side.

The muon number $N_{\mu}$ is very sensitive to $\alpha$. The relative increase in $N_{\mu}$ for a small change $\Delta \alpha$ is to first order

$$
\frac{\Delta N_{\mu}}{N_{\mu}} \approx \frac{\ln \left(E / \xi_{h}\right)}{\ln N_{\text {mult }}} \frac{\Delta \alpha}{\alpha}=k \frac{\Delta \alpha}{\alpha},
$$

where $k$ is the number of steps of the hadronic cascade. For a $\mathrm{EeV}$ air shower, $k \approx 5$, which implies that a $10 \%$ change in $\alpha$ introduces a $50 \%$ change in the muon number. Accordingly, $\alpha$ needs to be known rather precisely over a wide energy range to correctly calculate the muon number. In the simplified model, $\alpha$ is $2 / 3$ due to isospin symmetry, but the value differs in real air showers. 


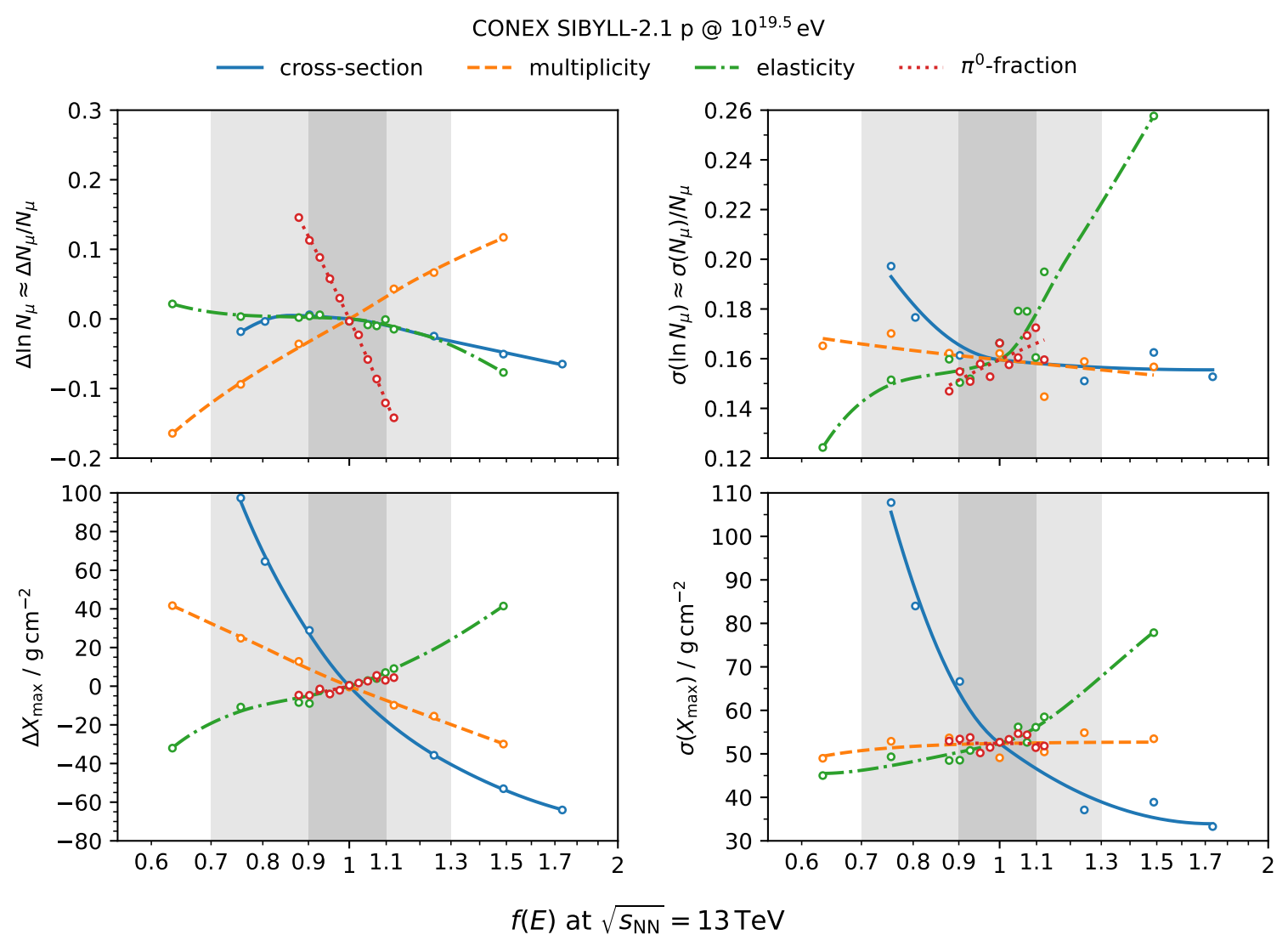

Figure 3: Impact of modifying the inelastic cross-section, the hadron multiplicity, the elasticity (energy fraction carried by the most energetic particle), and the fraction of neutral pions produced on the muon number $N_{\mu}$ and its fluctuations, as well as the depth of shower maximum and its fluctuations, for a proton shower with $10^{19.5} \mathrm{eV}$ simulated with SIBYLL-2.1 as the baseline. The modifications are shown as a function of the energy-dependent scale factor at the LHC energy scale of $13 \mathrm{TeV}$. Points represent the simulations results, line are empirical fits to guide the eye. Data from Ref. [16], image from Ref. [4].

Real air showers are more complex. Kaons, protons, neutrons, and strange hadrons are produced which have life-times large enough to participate in the cascade. The results of the previous calculations approximately carry over if $1-\alpha$ is considered more broadly as the energy fraction carried by neutral pions. Experimentally convenient is the closely related quantity

$$
R=\frac{E_{\mathrm{em}}}{E_{\mathrm{had}}},
$$

where $E_{\mathrm{em}}$ is the electromagnetic energy flow from photons and electrons, while $E_{\text {had }}$ is the hadronic energy flow, and the average is taken over the phase-space of the secondaries. The energy ratio $R$ is a function of pseudo-rapidity $\eta$ and its value at large $\eta$ is most important. The relationship to $\alpha$ is $R=(1-\alpha) / \alpha$, if $\alpha$ is considered as an energy fraction.

These analytical results were refined with full air shower simulations [16], in which basic features of hadronic interactions were modified ad-hoc with an energy-dependent scale factor to study the sensitivity of air shower observables on these features. The relevant results of this study 


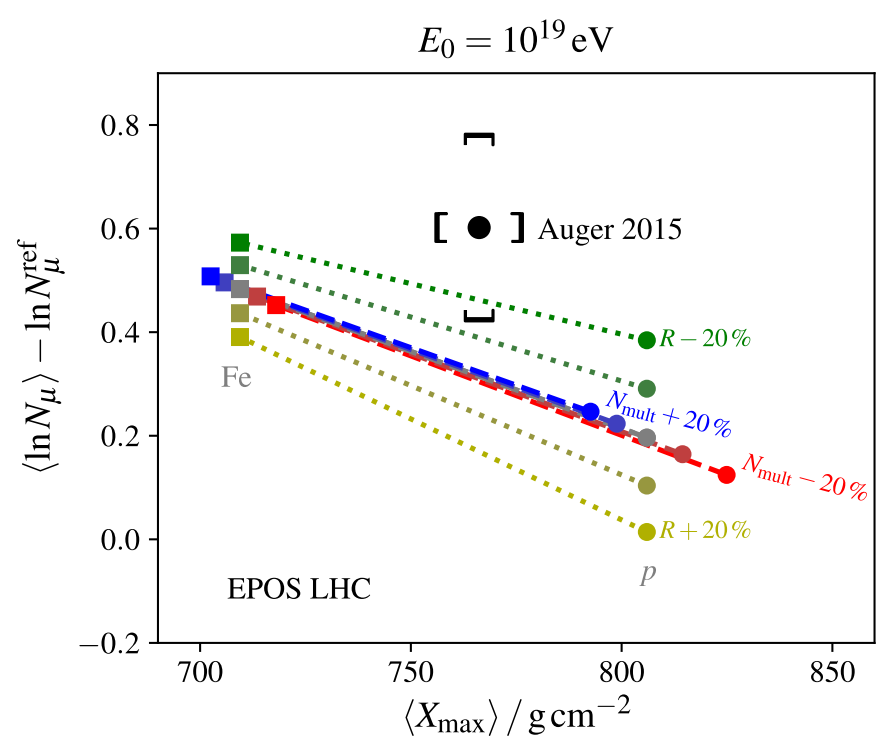

Figure 4: Impact of changing the hadron multiplicity and the energy ratio $\mathrm{R}=\left\langle E_{\mathrm{em}}\right\rangle /\left\langle E_{\mathrm{had}}\right\rangle$ on the muon number and the depth of shower maximum for $10^{19} \mathrm{eV}$ air showers. Lines indicate predictions from air shower simulations with EPOS-LHC [18] for any cosmic-ray composition between pure proton (bottom right) and iron (top left). The gray line indicates the baseline model, while the colored lines indicate predictions from an ad-hoc modified model, with modifications is steps of $10 \%$. The data point is from the Pierre Auger Observatory [19]. Image from Ref. [2].

are shown in Fig. 3. To increase the muon number in simulations one has to increase the hadron multiplicity and/or decrease the fraction of neutral pions produced. As predicted by the simplified model, the muon number is very sensitive to the fraction of neutral pions produced. It turns out that an increase in the hadron multiplicity changes the depth of shower maximum in such a way that the discrepancy cannot be resolved. This is shown in Fig. 4, the modified line is shifted parallel to itself, not closing the gap to the data point. The latter is only possible with a modification of $R$.

A reduction in the neutral pion fraction - or equivalent, a reduction in $R-$ is therefore the most plausible scenario, possibly accommodated by a moderate change in the hadron multiplicity $N_{\text {mult }}$.

\section{Related measurements at the LHC}

A comprehensive overview on existing LHC measurements that are relevant for air shower simulation is given in Ref. [4], we refer the interested reader to that paper for details and references, in the following we will give only an overview.

The LHC is mainly a proton-proton collider, but also proton-ion and ion-ion collisions are recorded. So far, protons, lead, and xenon ions have been accelerated. Important for air shower physics are the approved plans to accelerate of oxygen beams to measure $p-\mathrm{O}$ and $\mathrm{O}-\mathrm{O}$ collisions $[13,20]$ in 2023. The most common interaction in an air shower is $\pi-\mathrm{N}$ for which $p-\mathrm{O}$ collisions are an excellent reference. Current state-of-the-art models show considerable variance in their predictions of hadron production in $p$-O collisions, despite being tuned to $p p$ data, which reflects 

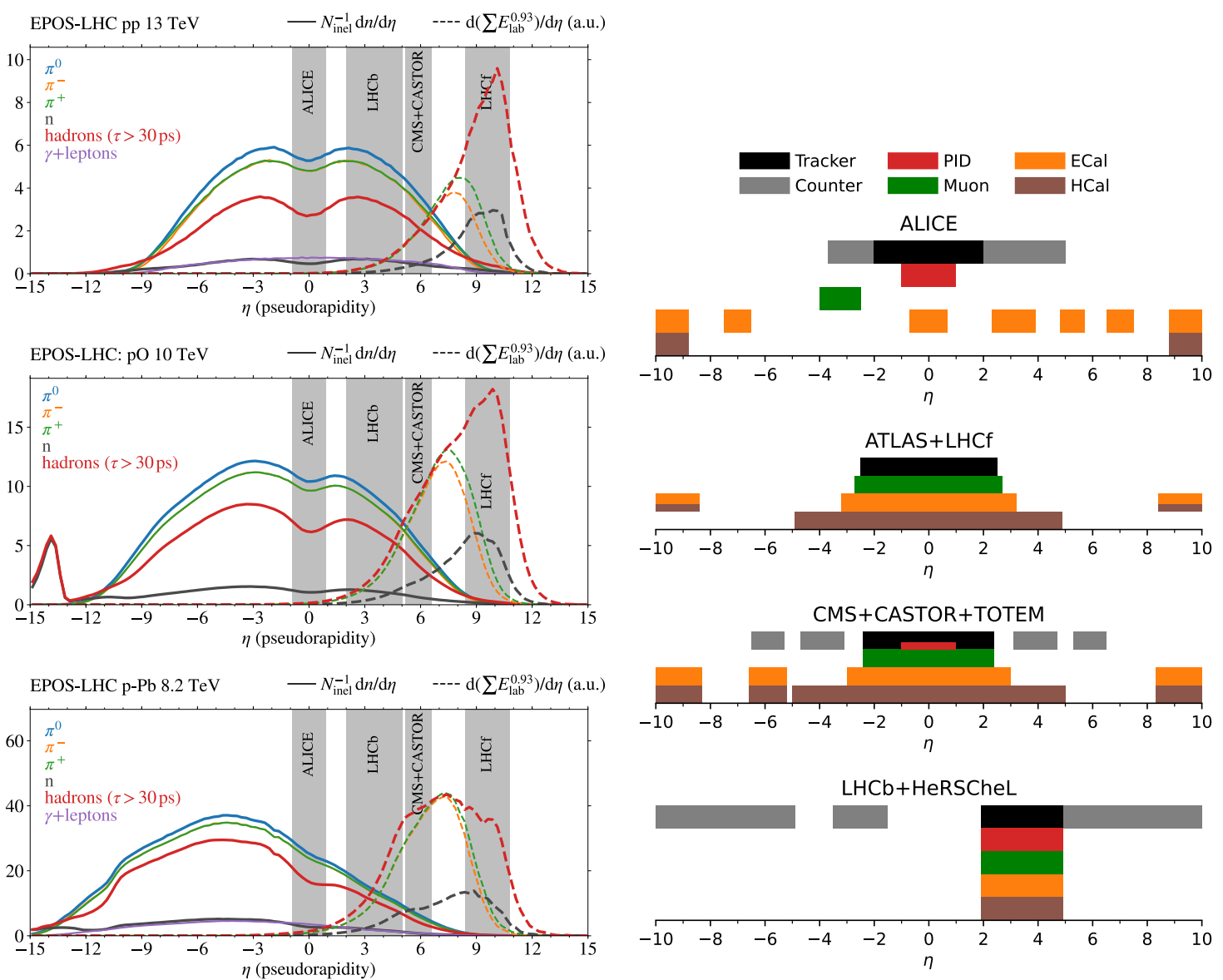

Figure 5: Left: Density of pions, neutrons, long-lived hadrons, photons and electrons as function of pseudorapidity $\eta$ in $p p, p-\mathrm{O}$, and $\mathrm{Pb}-\mathrm{Pb}$ collisions (solid lines). Shown for comparison is the expected muon yield from these particles if they form a shower in the atmosphere (dashed lines) and the acceptances of several LHC experiments (bands). Right: Acceptance of the LHC experiments and their detector components in pseudo-rapidity $\eta$. Counter refers to a detector that cannot measure the momentum of the particle, only its direction or local density; while a Tracker reconstructs the momentum and secondary vertices. PID refers to systems for particle identification; Muon to special muon trackers, and ECal, HCal to electromagnetic and hadronic calorimeters, respectively. Image taken from Ref. [4].

the theoretical uncertainties in extrapolating hadron production from a $p p$ reference system to a proton-ion collision. Together with the essential direct measurements in $p-\mathrm{O}$, the study of both $p p$ and $p-\mathrm{Pb}$ data is important to detect potential scaling laws and universal features of hadron-ion collisions.

A general challenge for air shower physics is that hadrons emitted in the forward region dominate the air shower development, but data are sparse in the forward region. This is shown in Fig. 5 on the left-hand side. The forward emitted particles are less numerous but more energetic and therefore create more secondary particles in the next interaction in the cascade. Eventually, the bulk of particles (and muons) is produced by the most energetic particles in the interaction.

As shown in Fig. 5 on the right-hand side, the LHC experiments are well instrumented at 
mid-rapidity $(|\eta|<2$ ), while the very forward region $|\eta|>5$ is only covered by calorimeters. There are technical and practical reasons for this, radiation levels are lower at mid-rapidity and particles can be accurately tracked even when the particle density is very high. LHCb is the most forward fully instrumented general purpose forward spectrometer, thanks to its focus on measuring decays of charm and beauty, and therefore of particular interest. Important in the very forward region are CMS with its CASTOR calorimeter system, the forward counters of TOTEM and ALICE, and the LHCf zero-degree calorimeters for photons and neutrons.

As discussed in the previous section, four basic aspects of hadronic interactions are relevant for air showers: the inelastic cross-section for hadrons in air, the hadron multiplicity, the elasticity (energy fraction carried by the most energetic particle), and finally the ratio $R$ electromagnetic to hadronic energy flow - or more generally speaking, the hadron composition.

The inelastic cross-section has a high impact on the predicted value of the depth of shower maximum $X_{\max }$ but not on the muon number $N_{\mu}$. It has been very accurately measured to a level of a few percent in proton-proton collisions at the LHC, in particular by TOTEM and ATLAS, which resolved the $1.9 \sigma$ ambiguity in earlier Tevatron data [21,22]. The CMS measurement of the $p-\mathrm{Pb}$ inelastic cross section [23] at $5.02 \mathrm{TeV}$ is also important, since it validated the standard Glauber model to better than about $10 \%$. This had a noted impact on the systematic uncertainty of $X_{\max }$ predictions. There is still a remaining uncertainty in the extrapolation of the inelastic cross-section from $p p$ to $p$-air, which can be reduced with future data from $p$-O collisions, but the inelastic cross-section is considered safe to extrapolate to higher energies and the model spread in this variable is very small.

The hadron multiplicity is important for both $X_{\max }$ and $N_{\mu}$. The experimental proxy for the hadron multiplicity is the charged particle multiplicity, which has been measured in $p p, p-\mathrm{Pb}$, $\mathrm{Pb}-\mathrm{Pb}$, and $\mathrm{Xe}-\mathrm{Xe}$ collisions at the LHC. Particularly important are the forward and very forward measurements from $\eta=2.5$ up to 6.4 by ALICE [25], LHCb [26], and TOTEM [27], because they constrain the hadron multiplicity in the important region for air showers. The shape of the hadron rapidity spectrum depends on the pomeron approach that is used in the hadronic models, and measurements over a wide pseudorapidity range are able to discriminate between the two main approaches in use, see Ref. [4] for details. As shown in Fig. 6 on the left-hand side, predictions of current models for $p p$ collisions deviate by less than $5 \%$ at $|\eta|<1$ because they are tuned to data at mid-rapidity, but the deviations in the forward region are of the order of 10 to $20 \%$. Recent data, not shown in Fig. 6, strongly constrains in the model predictions in the forward region in $p p$ collisions. A model variance of $20 \%$ is also found for $p-\mathrm{O}$ collisions, which is expected to be strongly reduced with the upcoming $p$-O data. Another experimental proxy for the hadron multiplicity is the energy flow, which has been measured in the forward direction by multiple experiments in $p p, p-\mathrm{Pb}$, and $\mathrm{Xe}$-Xe collisions, particular important for air showers are the forward and very forward measurements by LHCb and CMS with CASTOR, respectively. The accuracy of energy flow measurements is limited by the absolute calibration of the calorimeters, which tends to make the absolute measurements less precise than measurements based on charged particles.

The elasticity has an impact on $X_{\max }$, but not on $N_{\mu}$. It is difficult to measure, because the most energetic particle has a small angle to the beam and usually escapes detection. It can be measured with zero-degree calorimeters and has been constrained in $p p$ collisions with very-forward neutrons by LHCf [28]. Also important for the elasticity are the cross-sections for single- and double- 


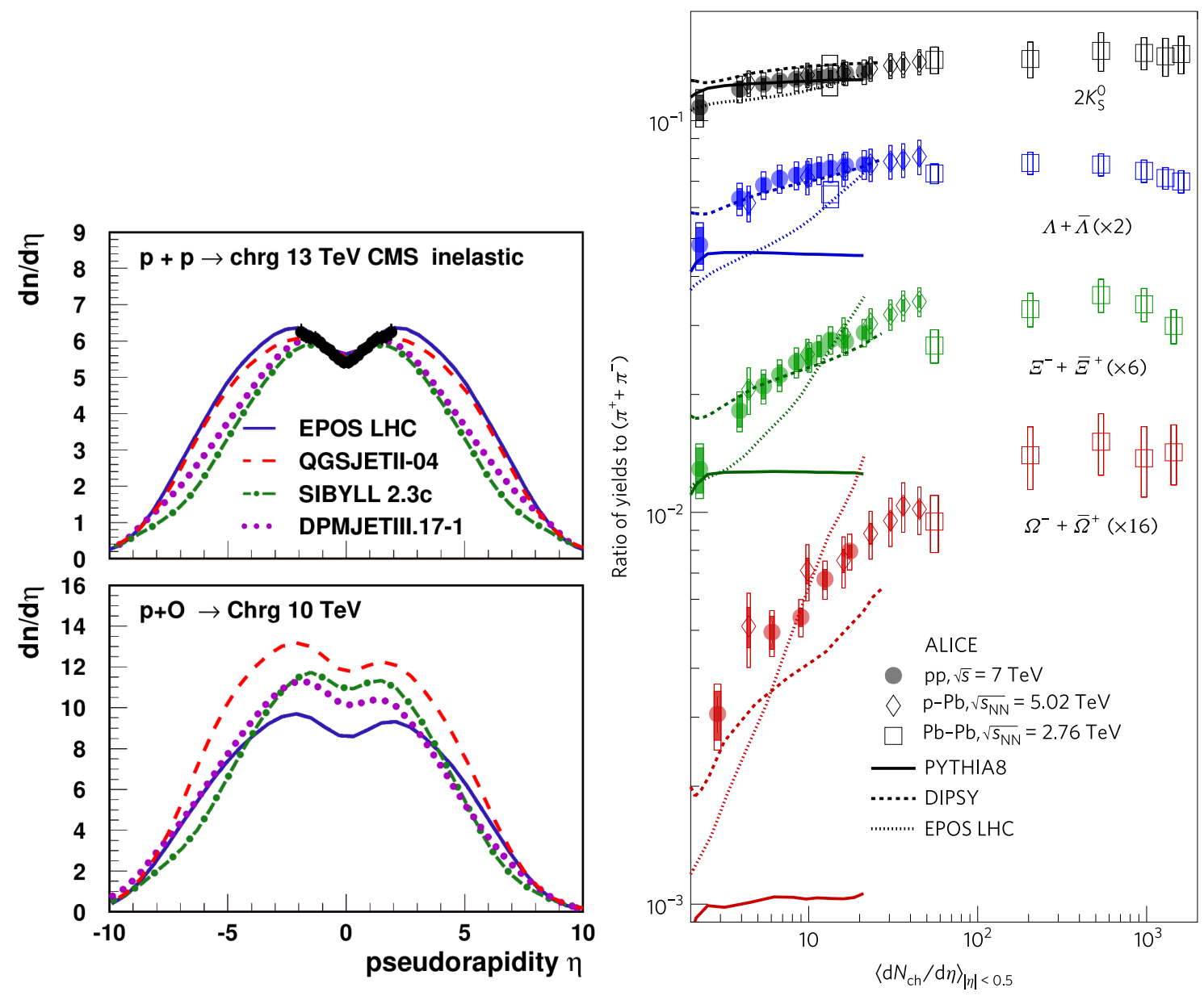

Figure 6: Left: Predictions of the charged particle density in minimum-bias pp collisions at $13 \mathrm{TeV}$ (top) and in unbiased $p-\mathrm{O}$ collisions at $10 \mathrm{TeV}$ (bottom). CMS data [24] are shown in the top plot for reference. Image from Ref. [4]. Right: Ratios of strange hadrons to pions measured at mid-rapidity $|\eta|<0.5$ in $p p$, $p-\mathrm{Pb}$, and $\mathrm{Pb}-\mathrm{Pb}$ collisions as function of the charged particle density $\mathrm{d} N_{\mathrm{ch}} / \mathrm{d} \eta$. Image taken from Ref. [1].

diffractive events, which make up about $20 \%$ of all inelastic events. In diffractive events, no color is exchanged between the beam particles, but particles are produced in the forward region from the fragmentation of color-neutral strings. Diffractive cross-sections have been studied in $p p$ collisions by ATLAS, CMS, ALICE, and TOTEM. Important are ongoing studies of the diffractive cross-section in $p-\mathrm{Pb}$ collisions to understand how they change in proton-ion collisions.

The hadron composition has the largest influence on $N_{\mu}$, but negligible impact on $X_{\max }$. Excellent high-precision data on the relative yields of pions, kaons, and protons in $p p, p-\mathrm{Pb}$, and $\mathrm{Pb}-\mathrm{Pb}$ collisions is available at mid-rapidity $|\eta|<1$ from ALICE and CMS. These data are important for model tuning and validation, but these measurements do not directly constrain the hadron composition in the forward region, where it matters for the muon production. In the forward region, yields of pions, kaons, and protons can only be studied by LHCb; other detectors lack the particle identification capabilities in this region. LHCb has published data from $p p$ collisions up to $7 \mathrm{TeV}$. Analysis of $p p$ collisions at $13 \mathrm{TeV}$ and $p-\mathrm{Pb}$ collisions at $8.16 \mathrm{TeV}$ are currently ongoing and expected to have an important impact on air showers. 
In the very forward region, the hadron composition was measured with the CASTOR calorimeter of CMS in $p p$ collisions, which provide a direct measurement of the ratio $R$ of electromagnetic to hadronic energy flow [2,29]. CMS found a high value of $R$ in $p p$ larger than what is predicted by all current models, but the value also has a rather large systematic uncertainty at the level of $-20 \%$ (the error is asymmetric) due to the challenging in-situ calibration of the calorimeter. The large value of $R$ in $p p$ found by CMS is surprising because it favors air shower simulations with even lower muon content than the current state-of-the-art, while the opposite is observed in air shower experiments. A follow-up study with $p-\mathrm{Pb}$ collision would be very important to understand whether $R$ is reduced in ion collisions compared to $p p$. A measurement of $p$-O collisions with CASTOR is unfortunately not possible, since CASTOR has been decommissioned and will not take data in 2023. In the very forward region, $R$ is constrained by LHCf with measurements of photon-production, $\pi^{0}$ production, and neutron production in $p p$ and $p$ - $\mathrm{Pb}$ collisions.

ALICE further studied the production cross-sections of strange hadrons at mid-rapidity $|\eta|<1$ and discovered an universal rise in the production ratios of strange hadrons to pions as a function of the charged particle multiplicity in the events in $p p, p-\mathrm{Pb}, \mathrm{Xe}-\mathrm{Xe}$, and $\mathrm{Pb}-\mathrm{Pb}[1,30]$, shown in Fig. 6 on the right-hand side. This behavior was previously known only from heavy-ion collisions and not expected in $p-\mathrm{Pb}$ and $p p$ collisions. Remarkable is the universality of the effect, the enhancement is the same in all systems and over the range of collision energies tested; it seems to depend only on the hadron multiplicity. The average charged particle density rises rapidly with the collision energy and thus the strangeness production in the average collision should rise as well. The strangeness enhancement is accompanied by a neutral pion reduction, which reduces $R$. The impact of this effect on air showers was studied phenomenologically with toy simulations. It was found that this modification has the potential to resolve the muon puzzle in air showers [2]. It is important to study this effect also in the forward region important for air showers, where several hadron production mechanisms contribute, string fragmentation and remnant fragmentation. Studying strange decays requires a full tracking system with vertex resolution and magnetic field, which currently only LHCb offers in the forward region. In the very forward region, CMS with CASTOR as studied $R$ as a function of the charged particle multiplicity at mid-rapidity [29], but found no significant trend. LHCf plans to study strangeness production at zero-degree angles based on the decay $K_{S}^{0} \rightarrow 2 \pi^{0} \rightarrow 4 \gamma$ with its upgraded detectors in the upcoming high-luminosity LHC run [31].

\section{Fixed-target experiments at SPS and LHC}

Since hadronic interactions in an air shower span over many orders of magnitude in energy, there are also opportunities to improve our knowledge at lower values of $\sqrt{s_{\mathrm{NN}}}$ reached by fixedtarget experiments [34]. One advantage of fixed-target experiments is the flexibility in regard to the target, which makes it ideal for studying nuclear effects in various systems.

The NA61/SHINE experiment at the SPS, the preaccelerator of the LHC, has measured hadron production in $p p, \pi-\mathrm{C}$, and $p-\mathrm{C}$ collisions, where carbon is used as a proxy for air. Among the many measurements, we highlight measurements of the forward $\rho^{0}$ and anti-proton production in Fig. 7. In those figures, the differential cross-section was integrated over the energy of the secondary particles to obtain the energy fraction carried by by $\rho^{0}$ s and anti-protons $[35,36]$. The 

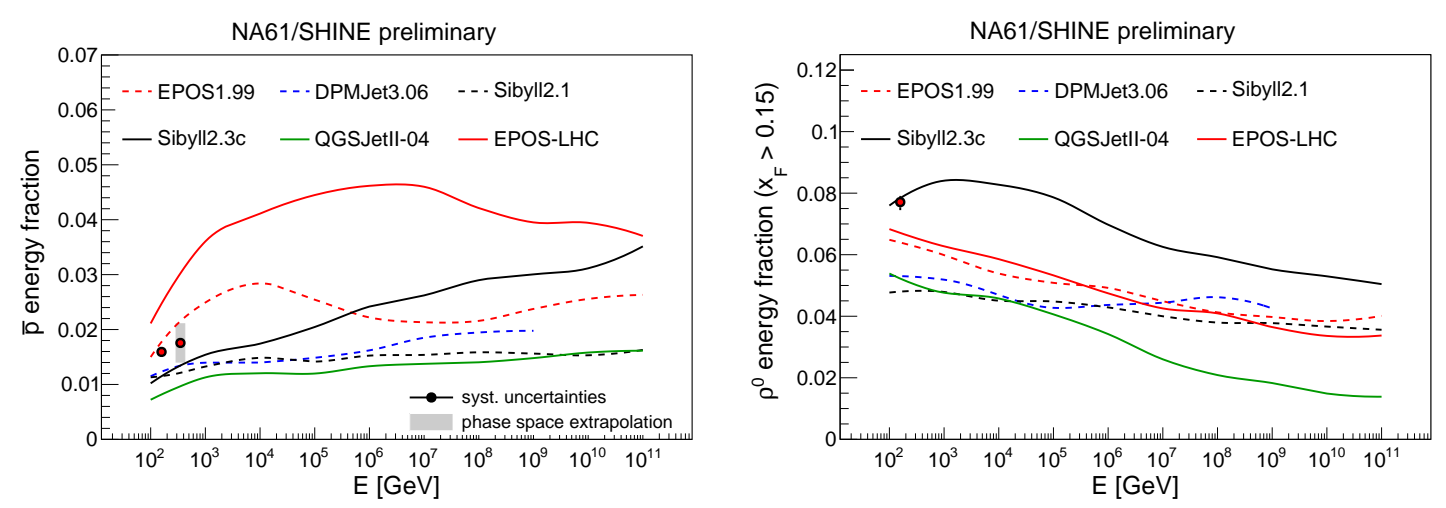

Figure 7: Energy fraction transferred to anti-protons (left) and $\rho^{0}$-mesons (right) in $\pi$-C collisions as measured by NA61/SHINE (data points) and as predicted by hadronic interaction models over the whole range of beam energies relevant for air showers (images from Refs. [32, 33]).

$\rho^{0}$ production is important since it is an alternative to producing a $\pi^{0}$ meson in the charge-exchange reaction $\pi^{-}+p \rightarrow \pi^{0}+n+X$. An enhancement of the $\rho^{0} / \pi^{0}$ ratio decreases $R$ and increases the muon number in air showers. Anti-protons are a measure of baryogenesis in the air shower, which reduces $R$. Although the energy flow towards these particle species is small in each interaction, the compounding effect over many interactions leads to an increase at the level of $60 \%$ in the muon number produced in air showers run with the recent version of SIBYLL-2.3d, which has these effects included, compared to SIBYLL-2.1, which has not [17]. These enhancements have not solved the muon puzzle, but it demonstrates the impact of studying the hadron composition also at lower energies.

At the LHC, fixed-target experiments are performed by LHCb with the SMOG device [37] that injects small amounts of gas into the detector. The original system was primarily designed to study the beam profile, but has been exploited to place limits on the intrinsic charm inside the proton [38] and to measure the anti-proton production cross-section in $p$-He collisions [39], which is needed to model cosmic ray interactions in space. The original device has been replaced as part of the LHCb upgrade for Run 3 with an open storage cell that allows for higher gas densities and more target gasses, including nitrogen and oxygen [40]. With LHCb in fixed-target mode, it will be possible to study hadron production at $\sqrt{s_{\mathrm{NN}}}=115 \mathrm{GeV} c^{-1}$ at mid-rapidity $-2.5<\eta_{\mathrm{cms}}<0.5$ in the nucleon-nucleon centre-of-mass frame.

\section{Summary and outlook}

The cosmic ray community has found a discrepancy in the muon production in air showers initiated by ultra-high energy cosmic rays. More muons are found than expected from air shower simulations. Air shower simulation codes model the hadronic cascade that gives rise to the $\mathrm{GeV}$ muons that are observable by surface arrays. The muons are produced at the end of this cascade in decays of pions and kaons, which means that hadron production needs to be modelled precisely over a wide energy range and in particular in the forward region by the hadronic interaction model used in the simulation. Studies of air shower simulations with modified hadronic interactions show that the most plausible way to increase the muon number is a modest reduction of the neutral pion 
fraction in the hadron composition over a wide range of collision energies. The onset of this effect should be observable already at the LHC. Also important are accurate measurements of the hadron multiplicity in the forward region.

To better understand hadron production in hadron-air collisions, measurements of protonproton collisions need to be complemented with proton-ion collisions to study nuclear effects. Important for air showers is the upcoming pilot run with proton-oxygen and oxygen-oxygen collisions at the LHC. LHC experiments with forward acceptance, ALICE, CMS, LHCb, LHCf and TOTEM, provide the most direct experimental constrains for air showers. $\mathrm{LHCb}$ is the only fully instrumented general purpose spectrometer with hadron identification capabilities in the forward region, while the other experiments provide calorimetric and particle counting information the in the very forward region.

Hadron production in proton-proton and proton-lead collisions is well explored at mid-rapidity. The charged particle density, an accurate proxy for the hadron multiplicity, has been measured over a wide range in pseudo-rapidity and now strongly constrains hadronic interaction models. In the forward region, more data from proton-lead collisions is needed to study nuclear effects and data from proton-oxygen collisions, which is underway. The discovery of a universal multiplicitydependent strangeness enhancement in proton-proton and proton-lead collisions at the LHC could be a potential solution to the muon puzzle. The effect was phenomenologically studied with toy simulations of air showers and found to potentially match the observed increase in muon production. The exact mechanism behind this effect is currently unclear, however. Further measurements of strangeness production with LHCb and LHCf are planned in the forward region, which is driving the hadron production in air showers. Further opportunities for air showers will be offered by running $\mathrm{LHCb}$ in fixed-target mode to the study collisions of the LHC beams with nitrogen and oxygen.

\section{References}

[1] ALICE collaboration, Enhanced production of multi-strange hadrons in high-multiplicity proton-proton collisions, Nature Phys. 13 (2017) 535 [1606 . 07424].

[2] S. Baur, H. Dembinski, M. Perlin, T. Pierog, R. Ulrich and K. Werner, Core-corona effect in hadron collisions and muon production in air showers, 1902.09265.

[3] T. Pierog, S. Baur, H. Dembinski, M. Perlin, R. Ulrich and K. Werner, When heavy ions meet cosmic rays: potential impact of QGP formation on the muon puzzle, in Proceedings of 37th International Cosmic Ray Conference - PoS(ICRC2021), vol. 395, p. 469, 2021, DOI.

[4] J. Albrecht et al., The Muon Puzzle in cosmic-ray induced air showers and its connection to the Large Hadron Collider, 2105. 06148.

[5] EAS-MSU, IceCube, KASCADE-Grande, NEVOD-DECOR, Pierre Auger, SugAR, Telescope ArRay, YAKUTSK EAS ARray collaboration, Report on Tests and Measurements of Hadronic Interaction Properties with Air Showers, EPJ Web Conf. 210 (2019) 02004 [1902.08124]. 
[6] EAS-MSU, IceCube, KASCADE Grande, NEVOD-DECOR, Pierre Auger, SUGAR, Telescope ARray, YAKUTSK EAS ARRAY collaboration, Working Group Report on the Combined Analysis of Muon Density Measurements from Eight Air Shower Experiments, PoS ICRC2019 (2020) 214 [2001.07508].

[7] D. Soldin, Update on the Combined Analysis of Muon Measurements from Nine Air Shower Experiments, in Proceedings of 37th International Cosmic Ray Conference PoS(ICRC2021), vol. 395, p. 349, 2021, DOI.

[8] J.H. Koehne, K. Frantzen, M. Schmitz, T. Fuchs, W. Rhode, D. Chirkin et al., PROPOSAL: A tool for propagation of charged leptons, Comput. Phys. Commun. 184 (2013) 2070.

[9] M. Dunsch, J. Soedingrekso, A. Sandrock, M. Meier, T. Menne and W. Rhode, Recent Improvements for the Lepton Propagator PROPOSAL, Comput. Phys. Commun. 242 (2019) $132[1809.07740]$.

[10] A. Sandrock, S.R. Kelner and W. Rhode, Radiative corrections to the average bremsstrahlung energy loss of high-energy muons, Phys. Lett. B 776 (2018) 350 [1706.07242].

[11] A. Sandrock and W. Rhode, Coulomb corrections to the bremsstrahlung and electron pair production cross section of high-energy muons on extended nuclei, 1807.08475.

[12] A. Fedynitch, R. Engel, T.K. Gaisser, F. Riehn and T. Stanev, Calculation of conventional and prompt lepton fluxes at very high energy, EPJ Web Conf. 99 (2015) 08001 [1503.00544].

[13] Z. Citron et al., Report from Working Group 5: Future physics opportunities for high-density QCD at the LHC with heavy-ion and proton beams, CERN Yellow Rep. Monogr. 7 (2019) 1159 [1812.06772].

[14] Pierre Auger collaboration, Measurement of the Fluctuations in the Number of Muons in Extensive Air Showers with the Pierre Auger Observatory, Phys. Rev. Lett. 126 (2021) 152002 [2102 . 07797].

[15] J. Matthews, A Heitler model of extensive air showers, Astropart. Phys. 22 (2005) 387.

[16] R. Ulrich, R. Engel and M. Unger, Hadronic Multiparticle Production at Ultra-High Energies and Extensive Air Showers, Phys. Rev. D 83 (2011) 054026 [1010 . 4310].

[17] F. Riehn, R. Engel, A. Fedynitch, T.K. Gaisser and T. Stanev, Hadronic interaction model Sibyll 2.3d and extensive air showers, Phys. Rev. D 102 (2020) 063002 [1912. 03300].

[18] T. Pierog, I. Karpenko, J.M. Katzy, E. Yatsenko and K. Werner, EPOS LHC: Test of collective hadronization with data measured at the CERN Large Hadron Collider, Phys. Rev. C 92 (2015) 034906 [1306.0121].

[19] Pierre Auger collaboration, Muons in Air Showers at the Pierre Auger Observatory: Mean Number in Highly Inclined Events, Phys. Rev. D 91 (2015) 032003 [1408 . 1421]. 
[20] R. Bruce, M.A. Jebramcik, J.M. Jowett, T. Mertens and M. Schaumann, Performance and luminosity models for heavy-ion operation at the CERN Large Hadron Collider, Eur. Phys. J. Plus 136 (2021) 745 [2107.09560].

[21] CDF collaboration, Measurement of the $\bar{p} p$ Total Cross-Section at $\sqrt{s}=546 \mathrm{GeV}$ and 1800 GeV, Phys. Rev. D 50 (1994) 5550.

[22] E710 collaboration, Measurement of $\rho$, the Ratio of the Real to Imaginary Part of the $\bar{p} p$ Forward Elastic Scattering Amplitude, at $\sqrt{s}=1.8-T e V$, Phys. Rev. Lett. 68 (1992) 2433.

[23] CMS collaboration, Measurement of the inelastic cross section in proton-lead collisions at $\sqrt{s_{N N}}=5.02 \mathrm{TeV}$, Phys. Lett. B 759 (2016) 641 [1509.03893].

[24] CMS collaboration, Pseudorapidity distribution of charged hadrons in proton-proton collisions at $\sqrt{s}=13 \mathrm{TeV}$, Phys. Lett. B 751 (2015) 143 [1507.05915].

[25] ALICE collaboration, Charged-particle multiplicity distributions over a wide pseudorapidity range in proton-proton collisions at $\sqrt{s}=0.9,7$, and $8 \mathrm{TeV}$, Eur. Phys. J. C 77 (2017) 852 [1708.01435].

[26] LHCв collaboration, Measurement of prompt charged-particle production in proton-proton collisions at a centre-of-mass energy of $13 \mathrm{TeV}, 2107.10090$.

[27] CMS, TOTEM collaboration, Measurement of pseudorapidity distributions of charged particles in proton-proton collisions at $\sqrt{s}=8 \mathrm{TeV}$ by the CMS and TOTEM experiments, Eur. Phys. J. C 74 (2014) 3053 [1405.0722].

[28] LHCF collaboration, Measurement of energy flow, cross section and average inelasticity of forward neutrons produced in $\sqrt{s}=13 \mathrm{TeV}$ proton-proton collisions with the LHCf Arm2 detector, JHEP 07 (2020) 016 [2003. 02192].

[29] CMS collaboration, Measurement of the average very forward energy as a function of the track multiplicity at central pseudorapidities in proton-proton collisions at $\sqrt{s}=13 \mathrm{TeV}$, Eur. Phys. J. C 79 (2019) 893 [1908.01750].

[30] ALICE collaboration, Strangeness production with ALICE at the LHC, Phys. Scripta 95 (2020) 064007.

[31] H. Menjo, O. Adriani, E. Berti, L. Bonechi, M. Bongi, R. D’Alessandro et al., Status and Prospects of the LHCf and RHICf experiments, in Proceedings of 37th International Cosmic Ray Conference - PoS(ICRC2021), vol. 395, p. 301, 2021, DOI.

[32] NA61/SHINE collaboration, Recent results from the cosmic ray program of the NA61/SHINE experiment, EPJ Web Conf. 208 (2019) 05006 [1810 . 00642].

[33] NA61/SHINE collaboration, New Results from the Cosmic-Ray Program of the NA61/SHINE facility at the CERN SPS, PoS ICRC2019 (2020) 446 [1909. 07136]. 
[34] C. Meurer, J. Bluemer, R. Engel, A. Haungs and M. Roth, Muon production in extensive air showers and its relation to hadronic interactions, Czech. J. Phys. 56 (2006) A211 [astro-ph/0512536].

[35] NA61/SHINE collaboration, Measurement of meson resonance production in $\pi^{-}+C$ interactions at SPS energies, Eur. Phys. J. C 77 (2017) 626 [1705 . 08206].

[36] NA61/SHINE collaboration, Measurements of Hadron Production in Pion-Carbon Interactions with NA61/SHINE at the CERN SPS, PoS ICRC2017 (2018) 315 [1707 . 07902].

[37] LHCв collaboration, Precision luminosity measurements at LHCb, JINST 9 (2014) P12005 [1410.0149].

[38] LHCв collaboration, First Measurement of Charm Production in its Fixed-Target Configuration at the LHC, Phys. Rev. Lett. 122 (2019) 132002 [1810 . 07907].

[39] LHCв collaboration, Measurement of Antiproton Production in $\mathrm{pHe}$ Collisions at $\sqrt{s_{N N}}=110 \mathrm{GeV}$, Phys. Rev. Lett. 121 (2018) 222001 [1808.06127].

[40] C. Barschel et al., LHC fixed target experiments : Report from the LHC Fixed Target Working Group of the CERN Physics Beyond Colliders Forum, vol. 4/2020 of CERN Yellow Reports: Monographs, CERN, Geneva (2020), 10.23731/CYRM-2020-004. 